\title{
RAILWAY CONDITION-BASED MAINTENANCE MODEL WITH STOCHASTIC DETERIORATION
}

\author{
Cecília VALE, Isabel M. RIBEIRO \\ Faculty of Engineering, University of Porto, Rua Dr. Roberto Frias s/n 4200-465, Porto, Portugal \\ Received 14 Jan 2012; accepted 10 Jul 2012
}

\begin{abstract}
The application of mathematical programming for scheduling preventive maintenance in railways is relatively new. This paper presents a stochastic mathematical model designed to optimize and to predict tamping operations in ballasted tracks as preventive condition-based maintenance. The model is formulated as a mixed 0-1 nonlinear program that considers real technical aspects as constraints: the reduction of the geometrical track quality over time is characterized by the deterioration rate of the standard deviation of the longitudinal level; the track layout; the dependency of the track recovery on its quality at the moment of the maintenance operation; the limits for preventive maintenance that depend on the maximum permissible train speed. In the model application, a railway stretch with $51.2 \mathrm{~km}$ of length is analysed for a time period of five years. The deterioration model is stochastic and represents the reduction of the standard deviation of the longitudinal level over time. The deterioration rate of the standard deviation of the longitudinal level is simulated by Monte Carlo techniques, considering the three parameters Dagum probabilistic distribution fitted with real data (Vale, Simões 2012). Two simulations are performed and compared: stochastic simulation in space; stochastic simulation in space and time. The proposed condition-based maintenance model is able to produce optimal schedules within appropriate computational times.
\end{abstract}

Keywords: condition-based maintenance model, stochastic deterioration, mixed 0-1 nonlinear programming, Monte Carlo technique.

\section{Introduction}

The track quality is guaranteed by performing conditionbased maintenance and renewal actions during the life of the track. These actions are of fundamental importance not only for the safe and efficient operation of trains on a railway track but also for passenger comfort. Preventive maintenance increases not only the system reliability but also its availability. However to minimize the resulting costs, scheduling maintenance operations, such as tamping, should be defined through optimization programs, because the correct cost estimation and the minimization of these operations contribute for the success of the railway project (Baalouska, Çelik 2011). The optimization problems may be solved by several types of algorithms. Some of them have already been applied for optimizing railway timetables, assigning locomotive at a minimal operational costs, optimizing networks, assigning extra trains on a railway network, etc. (Higgins et al. 1996; Rackwitz 2001; Lingaya et al. 2002; Kallen, Noortwijk 2005; Guan et al. 2006; Rouillon et al. 2006; Noortwijk 2009; Cacchiani et al. 2010).

The use of mathematical programming for scheduling preventive maintenance in railway is relatively new; however, some contributions on this theme considering different types of optimization models (Higgins et al. 1999; Budai et al. 2006; Oyama, Miwa 2006; Zhao et al.
2007; Vale et al. 2010) are emerging. Dekker (1996) presents an interesting review on applications of maintenance models. Optimization problems can be deterministic or probabilistic depending on the nature of the problem variables. In the first ones, no randomness or unceruncertainty is considered; in the others the uncertainties are taken into account by probabilistic distribution functions. The geometrical rail track deterioration is random by nature and it is usual to characterize that deterioration by the evolution over time (or tonnage) of several geometrical parameters such as the longitudinal level, the alignment, the gauge, the twist and the cross level.

The use of accurate methodologies for predicting the railway deterioration process increases maintenance effectiveness (Sadeghi, Askarinejad 2010). Therefore in this paper a stochastic mathematical model is designed to optimize and to predict tamping operations in ballasted tracks as preventive condition-based maintenance. The condition-based maintenance model is formulated as a mixed 0-1 nonlinear program with a stochastic deterioration model. An incomplete stochastic deterioration model for the longitudinal level (the geometrical parameters that most rapidly evolutes over time) is used. This model is considered to be incomplete because the data used for fitting consists of sparse observations of the deterioration process made over short time intervals. 


\section{Model formulation}

The proposed condition-based maintenance model for scheduling tamping on ballasted tracks takes into account the evolution over time of the track geometrical quality, the track layout influence of maintenance scheduling, the deterioration process over time, the dependency of the track geometrical quality recovery on its initial quality at the moment of maintenance operations and also the track quality limits that depend on maximum permissible vehicle speed. The track deterioration corresponds to the reduction of the standard deviation of the longitudinal level over time. The deterioration process is random by nature and it can be characterized by the Dagum probabilistic distribution as Vale and Simões (2012) illustrate.

\subsection{General description}

The main objective of the proposed model is to provide a methodology for optimal scheduling of preventive maintenance activities in a finite time horizon based on the track quality and on technical aspects related to tamping operations.

The model seeks an optimal solution corresponding to the minimum of the total number of tamping actions $M$ on a track for a predefined time horizon. If $m_{i j}$ is the binary variable associated to a preventive maintenance action on track segment $i$ and time period $j$, then the objective function takes the form expressed in Eqn (1):

$$
M=\min \sum_{i=1}^{n t} \sum_{j=1}^{n p} m_{i j},
$$

where: $n_{t}$ is the total number of track segments and $n_{p}$, the total number of time periods.

For the mathematical model formulation, some assumptions are taken into account:

a) the maintenance actions $m_{i j}$ correspond to tamping operations;

b) the deterioration rate of the standard deviation of the longitudinal level $\left(d_{i j}\right)$ is represented by a probabilistic distribution fitted with real data;

c) the irregularities of alignment, cross level, gauge and twist are disregarded;

d) the evolution of the standard deviation of the longitudinal level over time $\left(\sigma_{i j}\right)$ is defined by Eqn (2):

$$
\sigma_{i j}=\sigma_{i j-1}+d_{i j}-m_{i j} r_{i j}
$$

e) the recovery of the track quality $\left(r_{i j}\right)$ is linearly dependent on the value of the standard deviation of the longitudinal level of the track segment at the time of maintenance as shown in Eqn (3):

$$
r_{i j}=a\left(\sigma_{i j-1}+d_{i j}\right)+b,
$$

where: $a$ and $b$ are real parameters; $d_{i j}$ is a real parameter which represents the deterioration rate of the standard deviation of the longitudinal level on segment $i$ at time period $j ; r_{i j}$ is the recovery after maintenance of the standard deviation of the track longitudinal level on segment $i$ and time period $j$; $\sigma_{i j-1}$ is the standard deviation of longitudinal level on track segment $i$, at the period of time immediately previous to the maintenance operation; $\sigma_{i j-1}+d_{i j}$ is the standard deviation of longitudinal level at the time when maintenance is performed.

f) tamping operations begin and end on a straight alignment, according to UIC (2008) recommendations (Eqn 4):

$$
\sum_{l \in I(k)}^{n t} m_{l j} \geq|I(k)| m_{k j}
$$

with $k \in\left\{1, \ldots n_{t}\right\}, j \in\left\{1, \ldots n_{p}\right\}$ and $I(k) \subseteq\left\{1, \ldots n_{t}\right\}$ is the set of consecutive indexes of track segments that includes segment $k$ in curve and an initial and final segments in straight alignment.

According to Eqn (4), if a tamping operation is predicted for a curve segment, then the set of track segments that includes the curve segment (C) and it is bounded by straight segments (R) is also subjected to maintenance. Figure 1 represents this aspect.

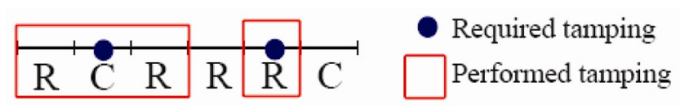

Fig. 1. Track layout influence on maintenance scheduling

\subsection{Problem formulation}

The mathematical formulation leads to the following mixed 0-1 nonlinear program:

$$
\begin{array}{ll}
\text { Minimize } & \sum_{i=1}^{n t} \sum_{j=1}^{n p} m_{i j} \\
\text { subject to }: & \sigma_{i j}=\sigma_{i j-1}+d_{i j}-r_{i j} m_{i j} \\
& r_{i j}=a\left(\sigma_{i j-1}+d_{i j}\right)+b \\
& \sum_{l \in I(k)}^{n p} m_{l j} \geq|I(k)| m_{k j} \\
& 0 \leq \sigma_{i j} \leq \sigma_{l i m} \\
& m_{i j} \in\{0,1\}
\end{array}
$$

with $i \in\left\{1, \ldots n_{t}\right\}, k \in\left\{1, \ldots n_{t}\right\}, j \in\left\{1, \ldots n_{p}\right\}$.

The existence of the bilinear function limits the use of commercial software in solving mixed integer problems, therefore a linearization of this program has be taken into account. This procedure is described in Vale et al. (2012) and consists of exploiting the so-called reformulation-linearization technique, proposed by Sherali and Adams (1999).

The application of the linearization to the problem (Eqn 5) leads one to the following mixed 0-1 linear programming problem. 


$$
\begin{array}{ll}
\text { Minimize } & \sum_{i=1}^{n t} \sum_{j=1}^{n p} m_{i j} \\
\text { subject to }: \quad & \sigma_{i j}=\sigma_{i j-1}+d_{i j}-w_{i j} \\
& r_{i j}=a\left(\sigma_{i j-1}+d_{i j}\right)+b \\
& 0 \leq w_{i j} \leq m_{i j} r_{\max } \\
& \left(1-m_{i j}\right) r_{\max } \leq r_{i j}-w_{i j} \leq\left(1-m_{i j}\right) r_{\max } \\
& \sum_{l \in I(k)}^{n t} m_{l j} \geq|I(k)| m_{k j} \\
& 0 \leq \sigma_{i j} \leq \sigma_{\lim } \\
& m_{i j} \in\{0,1\}
\end{array}
$$

The model parameters presented above are:

$-n_{t}$, total number of track segments;

$-n_{p}$, total number of discrete time periods (time horizon);

$-d_{i j}$, deterioration rate of the longitudinal level on the segment track $i$ at time period $j-(\mathrm{mm} / 90$ days) that follow the three parameters Dagum function, whose distribution function, $F(x)$, is defined by Eqn (7):

$$
F(x)=\left[1+\left(\frac{x}{\beta}\right)^{-\alpha}\right]^{-k}, \quad x>0 ;
$$

$-\sigma_{i 0}$, standard deviation of the longitudinal profile of the segment track $i$ at an initial time $j=0-(\mathrm{mm})$;

$-\sigma_{\text {lim }}$, limit for the standard deviation of the longitudinal profile depending on the vehicle maximum permissible speed - (mm);

$-a$ and $b$, real parameters;

$-I(k)$, set of consecutive indexes of track segments that include the segment $k$ in curve and an initial and final segments in straight alignment;

$-r_{\text {max }}$, maximum recovery of the standard deviation of the longitudinal level ( $\mathrm{mm})$;

$-|I(k)|$, number of elements of $I(k)$.

In this problem the decision variables are:

$-\sigma_{i j}$, standard deviation of the longitudinal level of the track segment $i$ at time period $j(\mathrm{~mm})$;

$-r_{i j}$, recovery of the standard deviation of the track longitudinal level on the segment $i$ at time period $j(\mathrm{~mm})$;

$-m_{i j}$, binary variable that denotes whether maintenance activity is assigned to the track segment $i$ at time period $j\left(\mathrm{~m}_{i j}=1\right)$ or not $\left(\mathrm{m}_{i j}=0\right)$;

$-\mathrm{w}_{i j}$, recovery after maintenance: $w_{i j}=r_{i j}$, if $m_{i j}=1$ or $w_{i j}=0$, if $m_{i j}=0$.

This mixed 0-1 linear program (6) leads to the same optimal solution as the mixed 0-1 nonlinear program (5) as presented.

Theorem: $(\bar{m}, \bar{\sigma}, \bar{r})$ is an optimal solution of the nonlinear problem (5) if and only if $(\bar{m}, \bar{\sigma}, r, \bar{w})$ is an optimal solution of the linear program (6), being $w$ a vector whose components are $w_{i j}=m_{i j} r_{i j}$.
Proof: As the objective functions of the two problems are equal, it is sufficient to prove that there is a one-to-one correspondence between the feasible solutions.

By defining vector $\bar{w}$, whose components are $\overline{w_{i j}}=\overline{m_{i j} r_{i j}}$, then, since $\overline{m_{i j}}$ is a binary variable (which takes values 0 or 1$)$, it follows that $(\bar{m}, \bar{\sigma}, \bar{r})$ is an optimal solution of problem (6) if and only if $(\bar{m}, \bar{\sigma}, \bar{r}, \bar{w})$ is an optimal solution of the mixed $0-1$ linear problem.

The proposed model (6) is a stochastic one being an extension of the deterministic model proposed by (Vale et al. 2012).

One of the features of this model is that it allows analysing the distribution of maintenance actions over time which is an advantage for evaluating maintenance costs over time. Therefore a mathematical programming approach for optimizing and scheduling maintenance may be a very useful tool for National Railway Administrations for the maintenance operations management.

\section{Computational experiments}

The proposed model is applied to a railway stretch of $51.2 \mathrm{~km}$ of length, defined by 256 track segments of $200 \mathrm{~m}$. The commercial program Cplex of the GAMS collection (Brooke et al. 1998) has been used to process the mixed-integer linear program defined in Eqn (6).

\subsection{Data}

The data used for testing the proposed model are described below and they were obtained based on real records measured in a railway line at regular time intervals.

- The standard deviation of the longitudinal level is calculated for track segments with $200 \mathrm{~m}$ of length. The standard deviation of the longitudinal level of each track segment at an initial time instant is represented in Figure 2. In this figure, the red line corresponds to the alert limit indicated in the European Standard EN 13848-5 (2008) for scheduling preventive maintenance actions. The alert limit depends on the maximum permissible speed. In this case, it is considered that the maximum speed in the railway stretch varies from 160 to $220 \mathrm{~km} / \mathrm{h}$.

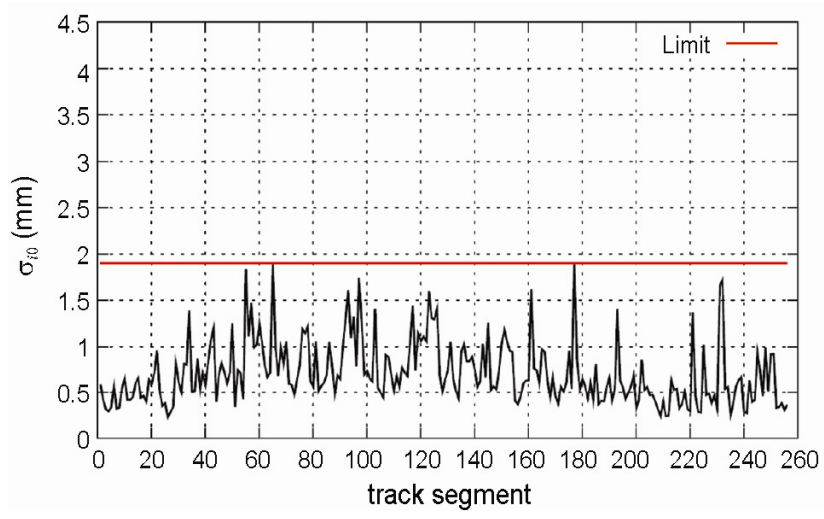

Fig. 2. Standard deviation of the longitudinal level of each track segment at the initial time instant 
- The deterioration rate of the standard deviation of the longitudinal level at each track segment is a random variable and it is characterized by the three parameters Dagum probabilistic distribution as described by Vale and Simões (2012). From the fitting process, the Dagum distribution is selected, because a good fit is achieved for representing the degradation of the standard deviation of the longitudinal level since the attained p-value of the KolmogorovSmirnov test is 0.66 . For this computational experience the adopted Dagum parameters fitted with real data are: $\alpha=2.3085 ; \quad \beta=0.0005582$ and $\mathrm{k}=0.50134$.

In this experiment, the deterioration rate of the standard deviation of the longitudinal level is simulated by Monte Carlo techniques, and two simulations are performed and compared:

A - stochastic simulation in space;

$\mathrm{B}$ - stochastic simulation in space and time.

In total, thirty calculations are executed for each one of the two simulations.

- The track layout is composed by straight and curve segments (Fig. 3).

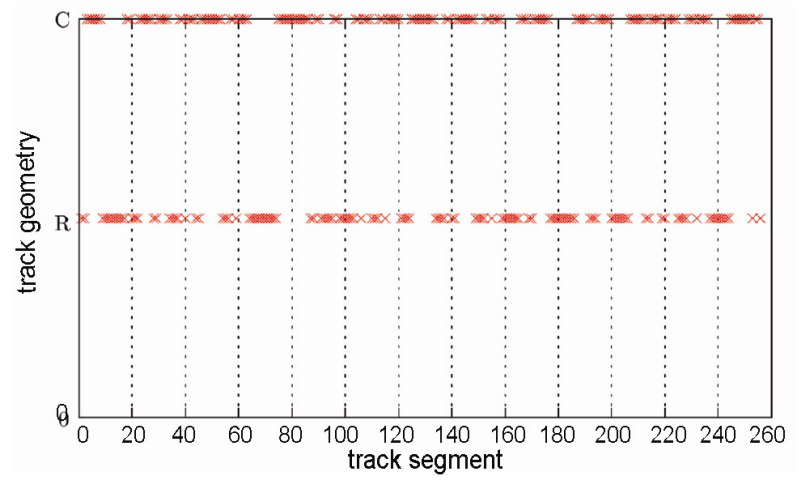

Fig. 3. Track layout ( $\mathrm{C}$ - curve; $\mathrm{R}$ - straight alignment)

The recovery of the standard deviation of the longitudinal level depends on the track geometrical quality at the moment of maintenance (ORE 1988). Although the recovery of the standard deviation of the longitudinal level is random as the deterioration rate of the standard deviation of the longitudinal level, in this research, the track recovery attained after tamping has been defined as a deterministic variable, based on real data measured in a railways line in order to reduce the computational effort in this computational experience:

$$
r_{i j}=0.4257 \sigma_{i j-1}-0.153 .
$$

- In this computational experience, a five year time horizon, defined by time instants of 90 days, is considered. This means that maintenance actions are scheduled quarterly.

\subsection{Results and discussion}

Figures 4 and 5 display, respectively, for simulations A and $\mathrm{B}$, the total number of maintenance actions and the total recovery associated to the optimal solution for a time horizon of five years. As previously referred, thirty scenarios are defined for each simulation.

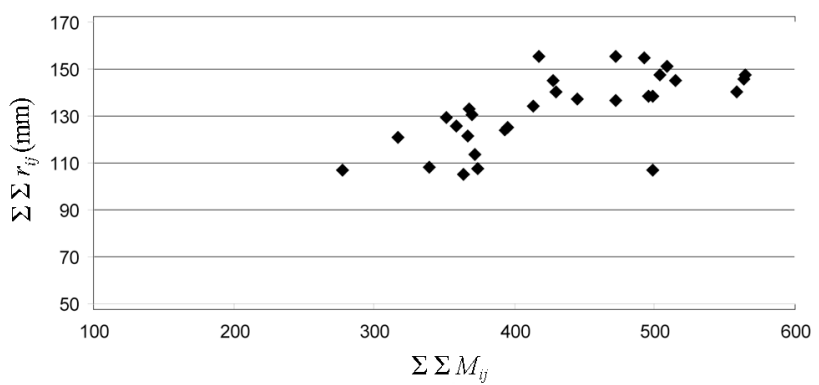

Fig. 4. Simulation A

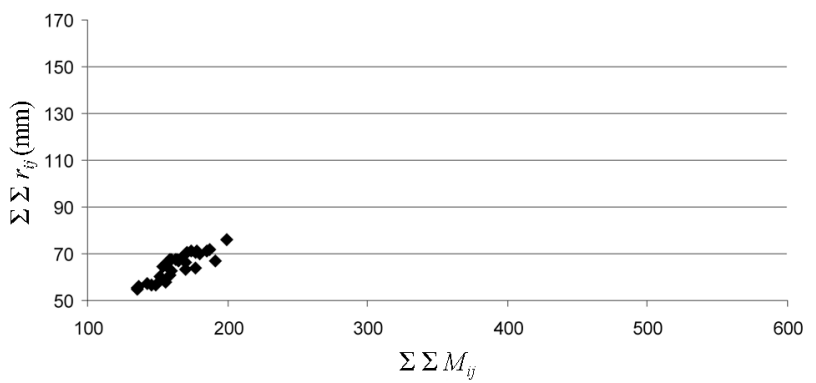

Fig. 5. Simulation B

From the figures, it is clear that the solutions in simulation $\mathrm{B}$ are more consistent than in simulation A, as also reckon by the standard deviation of the total number of maintenance actions $\left(\Sigma \Sigma M_{i j}\right)$ and the total track quality recovery $\left(\Sigma \Sigma r_{i j}\right)$ indicated in Table 1 . This table presents for a five years time horizon and the thirty scenarios: 1) a statistic description of the total number of maintenance actions; 2) the total track quality recovery and the ratio between the two parameters.

Table 1. Total number of maintenance actions and total track quality recovery

\begin{tabular}{lcccccc}
\cline { 2 - 7 } & \multicolumn{3}{c}{ Simulation $\mathrm{A}-d_{i}$} & \multicolumn{3}{c}{ Simulation $\mathrm{B}-d_{i j}$} \\
\cline { 2 - 7 } & $\Sigma \Sigma$ & \multirow{2}{*}{$\Sigma \Sigma r_{i j}$} & $\Sigma \Sigma r_{i j} /$ & $\Sigma \Sigma$ & \multirow{2}{*}{$\Sigma r_{i j}$} & $\Sigma \Sigma r_{i j} /$ \\
& $M_{i j}$ & & $M_{i j}$ & & $\Sigma \Sigma M_{i j}$ \\
\hline Mean & 431 & 132.343 & 0.313 & 164 & 64.910 & 0.397 \\
Maximum & 565 & 155.447 & 0.387 & 199 & 75.839 & 0.425 \\
Minimum & 277 & 105.035 & 0.214 & 135 & 54.818 & 0.351 \\
Standard & 77 & 15.715 & 0.041 & 16 & 5.795 & 0.018 \\
Deviation & & & & & & \\
\hline
\end{tabular}

The total number of maintenance actions is higher in simulation A than in B, which can be justified by the fact that the total deterioration of the standard deviation of the longitudinal level without considering any quality recovery $\left(\sum_{i=1}^{256} \sum_{j=1}^{20} d_{i j}\right)$ is higher in A than in B as indicated in Table 2.

As Noortwijk and Frangopol (2004) refer, the deterioration of the track longitudinal level is a random variable over time therefore simulation B represents a more realistic scenario. 
Table 2. Total deterioration of the standard deviation of the longitudinal level without maintenance

\begin{tabular}{|c|c|c|}
\hline & \multicolumn{2}{|c|}{$\sum_{i=1}^{256} \sum_{j=1}^{20} d_{i j}(\mathrm{~mm})$} \\
\hline & $d_{i}$ & $d_{i j}$ \\
\hline 1 & 243.9 & 50.4 \\
\hline 2 & 239.9 & 52.5 \\
\hline 3 & 218.1 & 51.0 \\
\hline 4 & 255.7 & 50.6 \\
\hline 5 & 236.2 & 53.2 \\
\hline 6 & 235.5 & 51.6 \\
\hline 7 & 243.0 & 53.1 \\
\hline 8 & 208.6 & 49.4 \\
\hline 9 & 197.9 & 48.8 \\
\hline 10 & 234.2 & 43.8 \\
\hline 11 & 234.7 & 48.8 \\
\hline 12 & 228.5 & 49.4 \\
\hline 13 & 205.9 & 50.0 \\
\hline 14 & 232.8 & 48.2 \\
\hline 15 & 228.2 & 49.4 \\
\hline 16 & 199.1 & 49.2 \\
\hline 17 & 234.6 & 48.2 \\
\hline 18 & 259.4 & 49.2 \\
\hline 19 & 244.0 & 51.1 \\
\hline 20 & 228.3 & 50.1 \\
\hline 21 & 231.1 & 53.5 \\
\hline 22 & 222.2 & 50.7 \\
\hline 23 & 251.9 & 49.1 \\
\hline 24 & 213.0 & 53.9 \\
\hline 25 & 250.5 & 53.0 \\
\hline 26 & 237.5 & 48.7 \\
\hline 27 & 251.7 & 46.9 \\
\hline 28 & 214.4 & 51.3 \\
\hline 29 & 251.3 & 52.7 \\
\hline 30 & 214.1 & 51.2 \\
\hline Mean & 231.5 & 50.3 \\
\hline Maximum & 259.4 & 53.9 \\
\hline Minimum & 197.9 & 43.8 \\
\hline $\begin{array}{l}\text { Standard } \\
\text { deviation }\end{array}$ & 16.8 & 2.2 \\
\hline
\end{tabular}

Table 3. Percentage of maintenance actions over the years

\begin{tabular}{|c|c|c|c|c|c|c|}
\hline & Simulation & $\begin{array}{c}\text { Year } \\
1\end{array}$ & $\begin{array}{c}\text { Year } \\
2\end{array}$ & $\begin{array}{c}\text { Year } \\
3\end{array}$ & $\begin{array}{c}\text { Year } \\
4\end{array}$ & $\begin{array}{c}\text { Year } \\
5\end{array}$ \\
\hline \multirow{2}{*}{ Mean } & A & 7.6 & 20.0 & 29.1 & 30.9 & 12.5 \\
\hline & B & 20.6 & 20.3 & 28.8 & 20.8 & 9.6 \\
\hline \multirow{2}{*}{ Maximum } & A & 15.0 & 25.7 & 39.7 & 47.3 & 17.7 \\
\hline & B & 38.3 & 39.7 & 57.3 & 35.2 & 28.2 \\
\hline \multirow{2}{*}{ Minimum } & A & 2.2 & 12.8 & 16.2 & 22.6 & 7.0 \\
\hline & B & 8.8 & 5.9 & 12.3 & 5.9 & 1.3 \\
\hline Standard & A & 3.0 & 2.9 & 4.6 & 5.5 & 3.3 \\
\hline deviation & $\mathrm{B}$ & 7.0 & 8.8 & 9.5 & 8.1 & 5.8 \\
\hline
\end{tabular}

Regarding the distribution over time of the maintenance actions, the percentage of those actions over the years is included in Table 3. The results show that the annual percentage of maintenance actions varies over the time horizon. In this case-study, a tamping cycle around 5 years is reckon, which is the period expected for this type of railway line.
For analysing the distribution of maintenance actions over time, several probabilistic distributions, such as the Exponential, the Gamma, the Weibull, the Logistic, the Lognormal, the Log-logistic, the Dagum, the Burr, etc. are tested. From the results, the distribution of maintenance actions over time (considering the 20 periods corresponding to 5 years time horizon) may be represented for both simulations by the Generalized Pareto Distribution, which probability density function is characterized by Eqns (8) and (9):

$$
f(x)= \begin{cases}1 / \sigma\left(1+k \frac{x-\mu}{\sigma}\right)^{-1-1 / k} & \text { if } k \neq 0 \\ 1 / \sigma \exp \left(-\frac{x-\mu}{\sigma}\right) & \text { otherwise }\end{cases}
$$

within the domain:

$$
\begin{array}{ll}
\mu \leq x<+\infty & \text { if } k \geq 0 \\
\mu \leq x \leq \mu-\sigma / k & \text { otherwise }
\end{array} .
$$

In Eqns (8) and (9), $k$ is a continuous shape parameter, $\sigma$ is a positive continuous scale parameter and $\mu$ is a continuous location parameter. The generalized Pareto distribution allows a continuous range of possible shapes that includes both the exponential $(k=0$ and $\mu=0)$ and Pareto distributions ( $k>0$ and $\mu=\sigma / k$, ) as special cases.

The Generalized Pareto distribution provides a good fit for the attained optimal solution as proven by the high p-value of the Kolmogorov-Smirnov test, except for the last time period (Table 4). In general the distribution fitting is better in simulation B than in A.

Table 4. P-value of the Kolmogorov-Smirnov test

\begin{tabular}{ccc}
\hline Time period & Simulation A & Simulation B \\
\hline 1 & 0,38335 & 0,38852 \\
2 & 0,57278 & 0,90445 \\
3 & 0,74915 & 0,88518 \\
4 & 0,49303 & 0,58197 \\
5 & 0,93664 & 0,95629 \\
6 & 0,81253 & 0,94015 \\
7 & 0,67287 & 0,67393 \\
8 & 0,4551 & 0,47944 \\
9 & 0,70271 & 0,79683 \\
10 & 0,71772 & 0,81192 \\
11 & 0,27263 & 0,50076 \\
12 & 0,43317 & 0,76877 \\
13 & 0,50514 & 0,49371 \\
14 & 0,71427 & 0,6911 \\
15 & 0,7149 & 0,69354 \\
16 & 0,29713 & 0,37983 \\
17 & 0,16404 & 0,27131 \\
18 & 0,41732 & 0,58294 \\
19 & 0,26348 & 0,30964 \\
20 & $\mathbb{0 , 0 1 6 4 5}$ & $\mathbf{0 , 0 3 1 7 7}$ \\
\hline
\end{tabular}


Table 5. Statistic analysis of the generalized Pareto distribution parameters

\begin{tabular}{lcccccc}
\hline & \multicolumn{3}{c}{$\mathrm{k}$} & \multicolumn{2}{c}{$\Sigma$} & \multicolumn{2}{c}{$\mu$} \\
\cline { 2 - 7 } & Sim. A & Sim. B & Sim. A & Sim. B & Sim. A & Sim. B \\
\hline Maximum & 0.397 & 0.394 & 27.242 & 24.266 & 2.029 & 1.667 \\
Mean & -0.254 & -0.253 & 10.841 & 11.844 & -0.695 & -0.765 \\
Minimum & -0.874 & -0.713 & -0.545 & 1.629 & -1.964 & -1.841 \\
Amplitude & 1.271 & 1.107 & 27.787 & 22.637 & 3.993 & 3.508 \\
Standard Deviation & 0.309 & 0.298 & 6.338 & 5.859 & 0.998 & 0.867 \\
\hline
\end{tabular}

From the statistic analysis of the generalized Pareto distribution parameters for the twenty time periods presented in Table 5, the parameters are similar for both simulations. However the values for simulation B are in general smaller than in A.

In terms of computation times, the proposed condition-based maintenance model is able to produce optimal schedules in a very short computational time as indicated in Table 6 which means that this model may be a useful tool for the maintenance operations management.

Table 6. Computation times

\begin{tabular}{lc}
\hline Mean & $32.369 \mathrm{~s}$ \\
Maximum & $276.351 \mathrm{~s}(4.6 \mathrm{~min})$ \\
Minimum & $3.015 \mathrm{~s}$ \\
Standard deviation & $57.361 \mathrm{~s}$ \\
\hline
\end{tabular}

\section{Summary and conclusions}

For defining condition-based maintenance tools that optimize the infrastructure asset management, the geometrical track deterioration has to be fully understood. The deterioration process is random by nature therefore it should be described by suitable probabilistic distribution functions fitted with real data.

This paper presents a preventive condition-based maintenance model for scheduling tamping in ballasted tracks considering stochastic deterioration. The model is formulated as a mixed 0-1 nonlinear program taking into account real technical aspects as constraints: the reduction of the geometrical track quality over time characterized by the deterioration rate of the standard deviation of the longitudinal level; the track layout influence on maintenance scheduling; the dependency of the quality recovery on the track quality at the moment of the maintenance operation; the limits for preventive maintenance that depend on the maximum permissible train speed. The random nature of the track deterioration process is characterized by a stochastic deterioration model based on the three parameters Dagum distribution function (Vale, Simões 2012).

In this research, two stochastic simulations and thirty scenarios in each simulation are performed. From the results, the total number of maintenance actions is higher in simulation $\mathrm{A}$ than in $\mathrm{B}$, because the total deterioration of the standard deviation of the longitudinal level without considering any quality recovery is higher in A than in B. Also the solutions in simulation $\mathrm{B}$ are more consistent than in simulation A.
As far as the model is concerned, it is able to produce useful results in terms of optimal schedules. The results attained in this model application show that the number of maintenance actions over time may be characterized by the Generalized Pareto distribution. From this analysis, one of the features of the proposed stochastic maintenance model is that it allows analysing the distribution of maintenance actions over time which is an advantage for evaluating maintenance costs during the life of the infrastructure. A mathematical programming approach for optimizing and scheduling maintenance such as the one presented in this paper may be therefore a very useful tool for National Railway Administrations for the maintenance operations management.

\section{Acknowledgements}

The present work has been funded by the Portuguese Foundation for Science and Technology (FCT).

\section{References}

Baalouska, Y.; Çelik, T. 2011. An integrated web-based data warehouse and artificial neural network system for unit price analysis with inflation adjustment, Journal of Civil Engineering and Management 17(2): 157-168. http://dx.doi.org/10.3846/13923730.2011.576806

Brooke, A.; Kendrick, D.; Meeraus, A.; Raman, R.; Borris, S. 1998. GAMS a user's guide. New York: GAMS Development Corporation. $316 \mathrm{p}$.

Budai, G.; Huisman, D.; Dekker, R. 2006. Scheduling preventive railway maintenance activities, Journal of the Operational Research Society 57: 1035-1044. http://dx.doi.org/10.1057/palgrave.jors.2602085

Cacchiani, V.; Caprara, A.; Toth, P. 2010, Scheduling extra freight trains on railway networks, Transportation Research Part B 44(2): 215-231. http://dx.doi.org/10.1016/j.trb.2009.07.007

Dekker, R. 1996. Applications of maintenance optimization models: a review and analysis, Reliability Engineering \& System Safety 51(3): 229-240. http://dx.doi.org/10.1016/0951-8320(95)00076-3

EN13848-5: 2008. Railway Applications - Track - Track geometry quality - Part 5: Geometric quality levels, European Committee for Standardization (CEN). $26 \mathrm{p}$.

Guan, J.; Yang, H.; Wirasinghe, S. 2006. Simultaneous optimization of transit line conguration and passenger line assignment, Transportation Research Part B 40(10): 885902. http://dx.doi.org/10.1016/j.trb.2005.12.003

Higgins, A.; Ferreira, L.; Lake, M. 1999. Scheduling rail track maintenance to minimise overall delays, in Proceedings of the $14^{\text {th }}$ International Symposium on Transportation and Traffic Theory, 20-23 July 1999, Jerusalem, Israel. 
Higgins, A.; Kozan, E.; Ferreira, L. 1996. Optimal scheduling of trains on a single line track, Transportation Research Part B 30(2): 147-161. http://dx.doi.org/10.1016/0191-2615(95)00022-4

Kallen, M. J.; Noortwijk, J. M. van. 2005. Optimal maintenance decisions under imperfect inspection, Reliability Engineering \& System Safety 90(2): 177-185. http://dx.doi.org/10.1016/j.ress.2004.10.004

Lingaya, N.; Cordeau, J.; Desaulniers, G.; Desrosiers, J.; Soumis, F. 2002, Operational car assignment at VIA Rail Canada, Transportation Research Part B 36(9): 755-778. http://dx.doi.org/10.1016/S0191-2615(01)00027-3

Noortwijk, J. M. 2009. A survey of the application of gamma processes in maintenance, Reliability Engineering \& System Safety 94(1): 2-21. http://dx.doi.org/10.1016/j.ress.2007.03.019

Noortwijk, J. M.; Frangopol, D. M. 2004. Two probabilistic life-cycle maintenance models for deteriorating civil infrastructures, Probabilistic Engineering Mechanics 19(4): 345-359. http://dx.doi.org/10.1016/j.probengmech.2004.03.002

ORE. 1988. Dynamic vehicle/track interaction phenomena from the point of view of track maintenance. Office for Research and Experiments of the International Union of Railways, Utrecht.

Oyama, T.; Miwa, M. 2006. Mathematical modelling analysis for obtaining an optimal railway track maintenance schedule, Japan of Industrial and Applied Mathematics 23(2): 207-224. http://dx.doi.org/10.1007/BF03167551

Rackwitz, R. 2001. Optimizing systematically renewed structures, Reliability Engineering \& System Safety 73(3): 269-279. http://dx.doi.org/10.1016/S09518320(01)00050-3
Rouillon, S.; Desaulniers, G.; Soumis, F. 2006. An extended branch-and bound method for locomotive assignment, Transportation Research Part B 40(5): 404-423. http://dx.doi.org/10.1016/j.trb.2005.05.005

Sadeghi, J.; Askarinejad, H. 2010. Development of improved railway track degradation models, Structure and Infrastructure Engineering 6(6): 675-688. http://dx.doi.org/10.1080/15732470801902436

Sherali, H.; Adams, W. 1999. A reformulation-linearization technique for solving discrete and continuous nonconvex problems. Boston: Kluwer Academic Publishers. 516 p. http://dx.doi.org/10.1007/978-1-4757-4388-3

UIC. 2008. Best practice guide for optimum track geometry durability, UIC Infrastructure Commission - Civil Engineering Support Group.

Vale, C.; Ribeiro, I.; Calçada, R. 2010. Application of a maintenance model for optimizing tamping on ballasted tracks: the influence of the model constraints, in Proceedings of the $2^{\text {nd }}$ International Conference on Engineering Optimization, 6-9 September 2010, Lisbon, Portugal.

Vale, C.; Ribeiro, I.; Calçada, R. 2012. Integer programming to optimize tamping in railway tracks as preventive maintenance, Journal of Transportation Engineering 138(1): 123-131. http://dx.doi.org/10.1061/(ASCE)TE.19435436.0000296

Vale, C.; Simões, M. L. 2012. Stochastic model for the geometrical rail track deterioration process, submitted into an international journal.

Zhao, J.; Chan, A. H. C.; Burrow, M. 2007. Reliability analysis and maintenance decision for railway sleepers using track condition information, Journal of the Operational Research Society 58: 1047-1055. http://dx.doi.org/10.1057/palgrave.jors.2602251

Cecília VALE. She is an Assistant Professor at the Transport Infrastructure Division in the Faculty of Engineering of the University of Porto. She has been involved in several research projects and she is author of some papers in international journals and conferences and reviewer of international journals. Her research interests are mainly in the field of railway engineering, dynamics and maintenance.

Isabel M. RIBEIRO. He is an Assistant Professor at the Mathematics Division of Department of Civil Engineering, Faculty of Engineering of the University of Porto. Research interests include global optimization, complementarity and applications of optimization in civil engineering. 